\title{
The potential insights of Traditional Chinese Medicine on treatment of COVID-19
}

\author{
Tong Tong ${ }^{\dagger}{ }^{\mathbb{D}}$, Ying-Qi Wu ${ }^{\dagger}$, Wei-Jian Ni, Ai-Zong Shen ${ }^{*}$ and Sheng Liü
}

\begin{abstract}
Corona Virus Disease 2019 (COVID-19) broke out in 2019 and spread rapidly around the world. There is still no specific antiviral therapy to the current pandemic. In China, historical records show that Traditional Chinese Medicine (TCM) is effective in prevention and enhancing the resistance to pandemic with unique insights. To fight with COVID-19, National Health and Commission of PRC has recommended some TCM in the guideline, such as HuoxiangZhengqi, LianhuaQingwen ShufengJiedu and XueBijing, and actually displayed a remarkable effect in clinical treatment strategic for COVID-19. We review studies to provide an in-depth understanding into the effect of TCM, and also introduce the possible mechanism involved in COVID-19 treatment.
\end{abstract}

Keywords: Traditional Chinese medicine, SARS-CoV-2, COVID-19, Treatment, Mechanism

\section{Background}

Currently, the disease caused by the Severe Acute Respiratory Syndrome Coronavirus 2 (SARS-CoV-2) was named Corona Virus Disease 2019 (COVID-19), have been spread around the world with over 2,430,000 confirmed cases and nearly 160,000 deaths (up to 21 April). Notably, the situation is getting worse and worse.

By comparing the infection patterns of coronavirus hosts in other vertebrates, SARS-CoV-2 was found to be similar to those of SARS-CoV and Middle East Respiratory Syndrome Coronavirus (MERS-CoV), SARS-CoV-2 could also be transmitted among humans. It can infect human cells by utilizing human angiotensin converting enzyme 2 (ACE2) as a receptor. Clinical presentation of COVID-19 is fever, fatigue, and dry cough and some patients show with nasal congestion, runny nose, inappetence, diarrhea and pneumonia on computed tomography $(\mathrm{CT})$. Some severe cases can rapidly develop into acute respiratory distress syndrome (ARDS), refractory

\footnotetext{
*Correspondence: 1649441800@qq.com; Islcclh|@163.com †Tong Tong and Ying-Qi Wu contributed equally to this work Department of Pharmacy, Anhui Provincial Hospital, The First Affiliated Hospital of USTC, Division of Life Sciences and Medicine, University of Science and Technology of China, Hefei 230001, Anhui, China
}

metabolic acidosis, septic shock, and coagulation dysfunction [1].

Unfortunately, there is no current any specific antiviral treatment for patients with suspected or confirmed COVID-19. According to the experiences in the SARS treatment, National Health Commission (NHC) of the PRC recommended TCM as strategies for COVID-19 treatment. From the current results, TCM has exhibited positive effects in combating with COVID-19. This article reviews focuses on the effects of 4 TCM in COVID19 treatment: HuoxiangZhengqi, LianhuaQingwen, ShufengJiedu and XueBijing, and summarize the mechanism of these drugs on COVID-19, to provide a deeper insight of therapeutic benefits of these TCM.

\section{HuoxiangZhengqi ameliorate symptoms through anti-inflammatory effects}

HuoxiangZhengqi (HXZQ) formula contains almost ten Chinese herbs. It is applied to traditional syndrome differentiation for cold, fever, nausea and vomiting, abdominal distension, diarrhea, also showed good effects in pediatric in dyspepsia to achieve the harmony inside and outside, the efficacy of antiemetic diarrhea. In NHC guidelines, HXZQ was recommended for clinical features

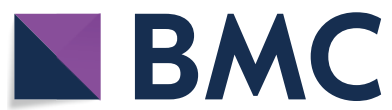

(c) The Author(s) 2020. This article is licensed under a Creative Commons Attribution 4.0 International License, which permits use, sharing, adaptation, distribution and reproduction in any medium or format, as long as you give appropriate credit to the original author(s) and the source, provide a link to the Creative Commons licence, and indicate if changes were made. The images or other third party material in this article are included in the article's Creative Commons licence, unless indicated otherwise in a credit line to the material. If material is not included in the article's Creative Commons licence and your intended use is not permitted by statutory regulation or exceeds the permitted use, you will need to obtain permission directly from the copyright holder. To view a copy of this licence, visit http://creativeco mmons.org/licenses/by/4.0/. The Creative Commons Public Domain Dedication waiver (http://creativecommons.org/publicdomain/ zero/1.0/) applies to the data made available in this article, unless otherwise stated in a credit line to the data. 
with aversion to cold with no sweating, headache, full chest diaphragm, urine frequency, yellow urine, etc.

According to the report from COVID-19 patients, the rise of creatine kinase $(\mathrm{CK})$ and lactate dehydrogenase (LDH) was said to be related to lung cell damage and systemic symptoms [1]. In SARS treatment, HXZQ showed good effects in improving $\mathrm{CK}, \mathrm{LDH}$ and oxygenation indexes [2]. It implied that HXZQ could improve oxygenation indexes and systemic symptoms through downregulating the level of $\mathrm{CK}$ and $\mathrm{LDH}$, might be a possible mechanism in treating COVID-19.

COVID-19 could cause strongly immune reaction. COVID-19 patients showed that in the peripheral blood inflammatory cytokines such as, IL-2, IL-6, IL-10 and Tumor Necrosis Factor $\alpha$ (TNF- $\alpha$ ) increased and $\mathrm{CD} 4^{+}, \mathrm{CD}^{+}, \mathrm{CD} 16^{+}, \mathrm{CD} 19^{+}$and $\mathrm{CD} 45^{+} \mathrm{T}$ cells were decreased [3], but an increase in Th17 cell proportion [4]. In animal model, HXZQ could regulate $\mathrm{CD}^{+}$and $\mathrm{CD}^{+}$cells and suppression on TNF- $\alpha$ level [5]. It indicates that HXZQ have the function of anti-inflammation and immune regulation in COVID-19 through suppress inflammatory factors and regulate immune response. In studies based on network pharmacology and molecular docking, researchers found that the core compounds of HXZQ such as quercetin, isorhamnetin, irisolidone, have a stronger binding ability to SARS-CoV-2 3CL (Mpro) than that of remdesivir with COVID-19. They could combine with ACE2 binding to PI3K-Akt signaling pathway to affect viral replication, thus exerting therapeutic effect on COVID-19. Which is worthy of further research and helps to provide theoretical guidance [6].

\section{LianhuaQingwen protect lung from pneumonia via inhibiting pro-inflammatory cytokines production}

LianhuaQingwen (LHQW) formula is composed of 13 Chinese herbs, which was approved in the SARS treatment in 2003. It has become an effective treatment for SARS-CoV, MERS-CoV, $\mathrm{H}_{1} \mathrm{~N}_{1}, \mathrm{H}_{3} \mathrm{~N}_{2}$, and $\mathrm{H}_{7} \mathrm{~N}_{9}$. Analysis of COVID-19 treatment with LHQW indicate that LHQW could significantly relieve clinical symptoms in patients with fever, weakness, cough and reduce the course of the COVID-19 [7]. The molecular docking results showed that the key components are kaempferol, quercetin, luteolin, glycyrrhetinic acid, stigmasterol, indigo had good binding ability with SARS-CoV-2 3CL (Mpro) and ACE2, acts on COVID-19 through multiple components, multiple targets, and multiple pathways [8].

Lung, which is the target organ of COVID-19, according to the TCM theory, "damp and toxin plague" is the main cause of COVID-19 etiology, even cause fatal pneumonia. Increasing evidence points out that cytokine storm displays a key role in causing fatal pneumonia [9].
In pulmonary oxidative lesions models, LHQW could significantly reduce pathological changes, including alveolar septum thickening, capillary congestion, interstitial edema, peripheral bronchial lymphocyte infiltration and neutrophils, the mechanism might be related to the levels of malonaldehyde, LDH, glutathione peroxidase, and super oxide dismutase were regulated by LHQW, which play significant role in pathogenesis of lung injury [10]. LHQW could inhibits the replication of SARS-COV-2 in vitro, and significantly reduced pro-inflammatory cytokines production (IL- 6 , TNF- $\alpha$ ), which might mediate strong immune response or even cytokine storm [11].

In children with mycoplasma pneumoniae pneumonia, after treatment with $\mathrm{LHQW}$, the $\mathrm{CD}^{+}, \mathrm{CD} 4^{+}$, and $\mathrm{CD}^{+} \mathrm{T}$ cell subsets in patients were significantly altered, and IL-6, c-reactive protein (CRP) in serum and procalcitonin (PCT) levels significantly reduced [12]. In COVID19 patients treat with LHQW, the total effective rate was $74.55 \%$, and 28 patients were cured after 3 days. After 7 days of treatment, the total effective rate was $92.73 \%$ and 39 patients were totally cured, main symptoms patients experienced including fever, cough, fatigue and chest tightness were significantly reduced [7].

\section{ShufengJiedu act on COVID-19 through multiple targets and multiple inflammatory signaling pathways}

The main components of ShufengJiedu (SFJD) are polygonum cuspidatum, forsythia, radix isatidis, bupleurum root, rhizoma corydalis, verbena, reed root, liquorice. Previous research suggested that SFJD can alleviate the clinical symptoms of patients with Acute Exacerbation of Chronic Obstructive Pulmonary Disease (AECOPD) and shorten the hospitalization time [13]. SFJD not only had the function of inhibiting virus proliferation and antiinflammatory, but also has certain immune regulation function. Whatever in vivo or vitro, SFJD had a function of inhibiting airway inflammatory responses via regulating NLRP3 inflammasome and then down-regulating the level of IL-18 and IL-1 $\beta$ which similar to these effects of oseltamivir [14].

SFJD combined with western medicine treatment in COVID-19 have been gained significant improvement in pneumonia associated symptoms [15]. The combination of SFJD and Arbidol was better than Arbidol alone in the treatment of COVID-19, which could significantly shorten the symptoms improvement time and negative conversion time of the clinical patients [16]. In deeper studies, after SFJD treatment, partial pressure of oxygen in lung tissue increased, the level of lactic acid decreased, inflammatory cytokines such as IL-1 $\beta$, IL- 6 and TNF- $\alpha$ were inhibited [17]. Latest molecular docking results showed that quercetin, kaempferol, luteolin, these core 
compounds in SFJD had high affinity with target proteins. Similar to LHQW, these chemical compounds involved a variety of biological processes and pathways to treat COVID-19 by combining with key target proteins IL-6, ALB, and MAPK3, which supported the clinical application with COVID-19 [18].

\section{XueBijing injection reduce multiple organ damage caused} by COVID-19 through anti-inflammation and improving immune function

With the approving for marketing in 2004 by Chinese authorities, Xuebijing (XBJ) injection has been used in H1N1, H7N9, dengue fever, MERS as well as ebola. From previous report, XBJ could antagonize endotoxin, anti-inflammation, improving immune function and microcirculation, and regulating coagulation disorders $[19,20]$. COVID-19 patients often occur respiratory distress, coagulation disorders and microcirculation disorders, especially in patients with systemic inflammatory response syndrome or/and multiple organ failure, timely use of XBJ can effectively reverse the situation and reduce the fatality. Currently, Chinese researchers are now conducting a prospective analysis of the clinical efficacy of XBJ on COVID-19. Hydroxysafflor yellow $\mathrm{A}$, chlorogenic acid and salvianolic acid $\mathrm{B}$ were major compositions in XBJ by molecular docking [21], through "multi-component, multi-target, multi-pathway" to play the role of anti-inflammatory, vascular endothelial protection and immunity. XBJ could inhibit inflammatory cytokines such as IL-1, IL-6, IL-8, IL-17 and TNF- $\alpha$ [22]. By increasing the Th1/Th2 ratio, XBJ injection could improve the proportion of Th1 cells in septic rats [23], promote the apoptosis of $\mathrm{CD} 4^{+} \mathrm{CD} 25^{+} \mathrm{T}$ cells (Tregs) $[24,25]$, and further improve the immune function.

\section{Potential mechanism of 4 TCM in COVID-19 treatment}

To date, NHC has issued 7 editions guidelines of diagnosis and treatment for COVID-19. In each edition, TCM has been recommended for COVID-19 treatment based on different stage and symptom differentiation. TCM has shown good effects in combating with COVID-19, early intervention of TCM in COVID-19 treatment could increase cure rate, shorten disease course and reduce mortality cases. According to the guidelines, 4 TCM in this paper and main ingredients and traditional indications versus COVID-19 are as follow (Tables 1, 2).

So far as we know, COVID-19 could cause mortal systemic complication with strongly immune response or
Table 14 TCM recommended by guidelines of treatment for COVID-19

\begin{tabular}{lll}
\hline & Stage of disease & Symptom \\
\hline HXZQ & Medical observation period & $\begin{array}{c}\text { Hypodynamia with gastrointestinal } \\
\text { upset }\end{array}$ \\
LHQW & Medical observation period & Hypodynamia with fever \\
SFJD & Medical observation period & Hypodynamia with fever \\
XBJ & Clinical treatment period & Several cases and critical cases \\
\hline
\end{tabular}

cytokine storm, further cause multiple organ dysfunction syndrome (MODS), which is the main cause of mortality in COVID-19 (Fig. 1).

TCM can regulate the inflammatory response of the body through "multi-component, multi-target, multipathway" to improve the immunity of the body, so as to ameliorate symptoms, prevent complications, and achieve indirect suppression of the virus. Through the prediction of molecular docking, major chemical constituents and possible targets of 4 TCM in COVID-19 were found (Table 3). According to the analysis and illumination from the latest literatures, we summarized possible mechanism and related targets of LHQW in treating with COVID-19 and showed it in Fig. 2.

\section{Conclusion}

With the emergence of COVID-19, three cases of zoonotic coronavirus disease have been identified in this century. However, COVID-19 has caused more deaths to date than SARS and MERS. Accumulated experiences from thousands of years in the treatment of epidemic, TCM is worth learning. TCM has holistic therapy theory, it could balance Yin and Yang, enhancing human body resistance to eliminate epidemic factors. That's why China government recommended TCM in combating COVID-19 timely.

Although, laboratory studies on the effect of TCM are far behind the clinical application in COVID-19 treatment, further studies in molecular mechanisms are expected to clarify the effect of TCM on COVID-19. In this study, combining with the latest research, this work highlights the prospect of therapeutic effects and mechanism of 4 TCM in COVID-19 treatment. The therapeutic effects of 4 TCM in COVID-19 potentially focus on: anti-inflammatory, inhibiting pro-inflammatory cytokine production and cut off cytokine storm, regulating immune response, protecting organ damage. By the continuing expansion of this pandemic, we anticipate more 
Table 2 Main ingredients and traditional indications versus COVID-19

\begin{tabular}{|c|c|c|c|}
\hline & Main ingredients & Traditional indications & COVID-19 \\
\hline HXZQ & $\begin{array}{l}\text { Ageratum, poriacocos, perilla, angelica, } \\
\text { orange peel, radix platycodonis, atracty- } \\
\text { lodes, magnolia officinalis, pinellia, liquorice }\end{array}$ & $\begin{array}{l}\text { Gastrointestinal cold, influenza and upper } \\
\text { respiratory tract infections, viral enteritis, } \\
\text { diarrhea }\end{array}$ & $\begin{array}{l}\text { Hypodynamia accompanied by gastrointestinal } \\
\text { upset, cold without sweating, headache and } \\
\text { heaviness, limb pain, thirst with no desire to } \\
\text { drink, yellow urine, frequent micturition }\end{array}$ \\
\hline LHQW & $\begin{array}{l}\text { Forsythia, honeysuckle, ephedra, male fern } \\
\text { rhizome, houttuyniae, pogostemon cablin, } \\
\text { rheum, rhodiola, menthol, liquorice }\end{array}$ & $\begin{array}{l}\text { Fever, aversion to cold, muscular soreness, } \\
\text { stuffy nose runny nose, cough, head- } \\
\text { ache, pharyngoxerosis and pharyngalgia }\end{array}$ & Hypodynamia and fever \\
\hline SFJD & $\begin{array}{l}\text { Polygonum cuspidatum, forsythia, isatidis, } \\
\text { bupleurum, rhizoma corydalis, verbena, } \\
\text { reed root, liquorice }\end{array}$ & $\begin{array}{l}\text { Fever, aversion to wind, pharyngalgia, } \\
\text { headache, stuffy nose runny nose, } \\
\text { cough }\end{array}$ & Hypodynamia and fever \\
\hline XBJ & $\begin{array}{l}\text { Paeoniae, angelica rhizoma Chuanxiong, flos } \\
\text { carthami, salviae miltiorrhizae }\end{array}$ & $\begin{array}{l}\text { Fever, dyspnea, palpitation, dysphoria, } \\
\text { infection, viscera damage }\end{array}$ & $\begin{array}{l}\text { Dyspnea, high fever or alternating cold and heat, } \\
\text { cough with less phlegm, coma, etc. }\end{array}$ \\
\hline
\end{tabular}

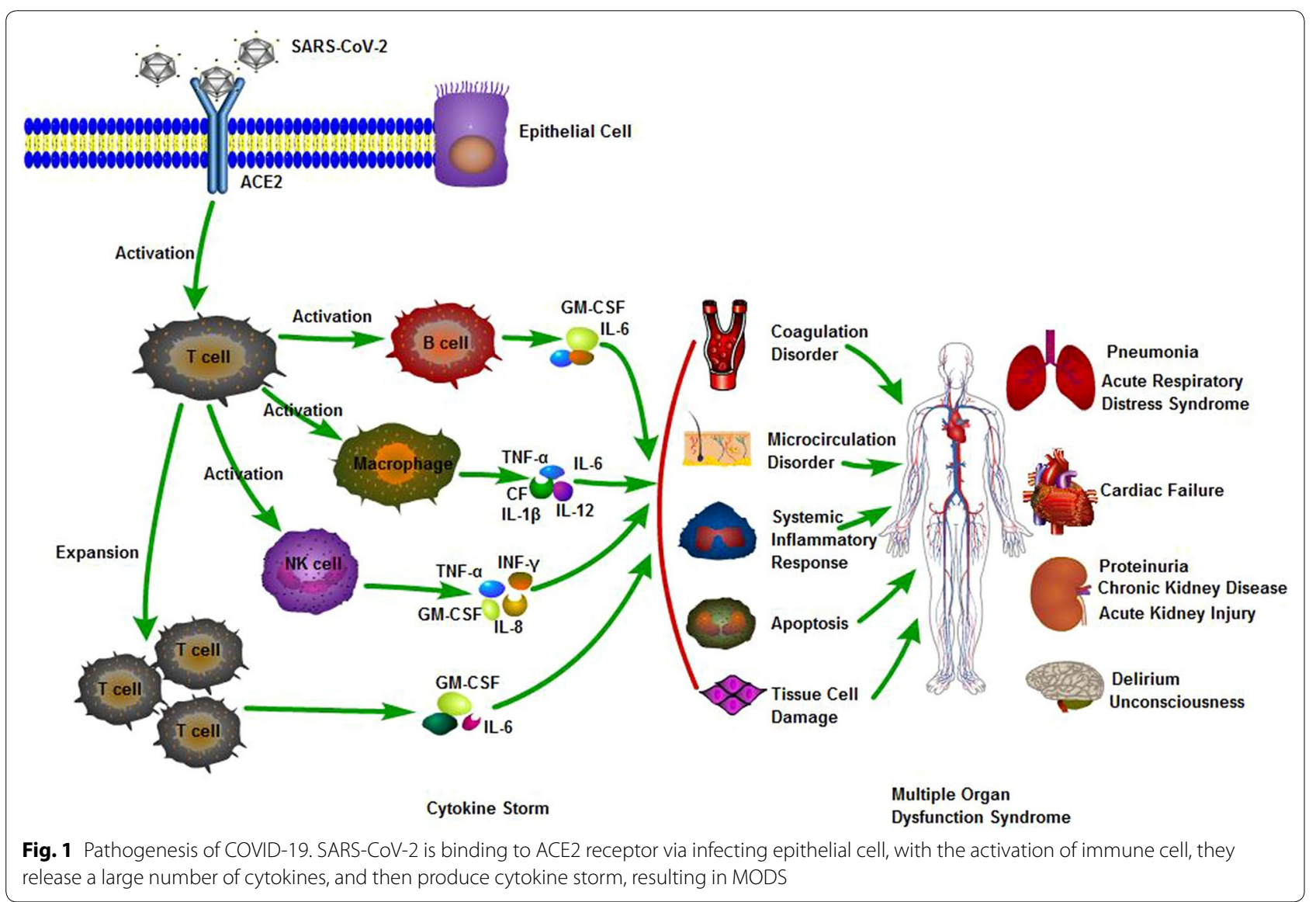

Table 3 Major chemical constituents and possible targets of TCM in COVID-19

\begin{tabular}{llll}
\hline & Major chemical constituents & Possible targets & References \\
\hline HXZQ & Quercetin, isorhamnetin, irisolidone, kaempferol, wogonin, baicalein & PTGS2, HSP90AB1, CAMSAP2 & [5, 6] \\
LHQW & Kaempferol, quercetin, glycyrrhetinic acid, stigmasterol, indigo & SARS-CoV-2 3CL (Mpro), ACE2, MAPK, & {$[8]$} \\
& & PI3K-AKT, NF-KB & IL-6, ALB, MAPK3 \\
SFJD & Quercetin, kaempferol, luteolin & NF-KB, HIF-1, VEGF & {$[18]$} \\
XBJ & Hydroxysafflor yellow A, chlorogenic acid, salvianolic acid B & & {$[21]$} \\
\hline
\end{tabular}




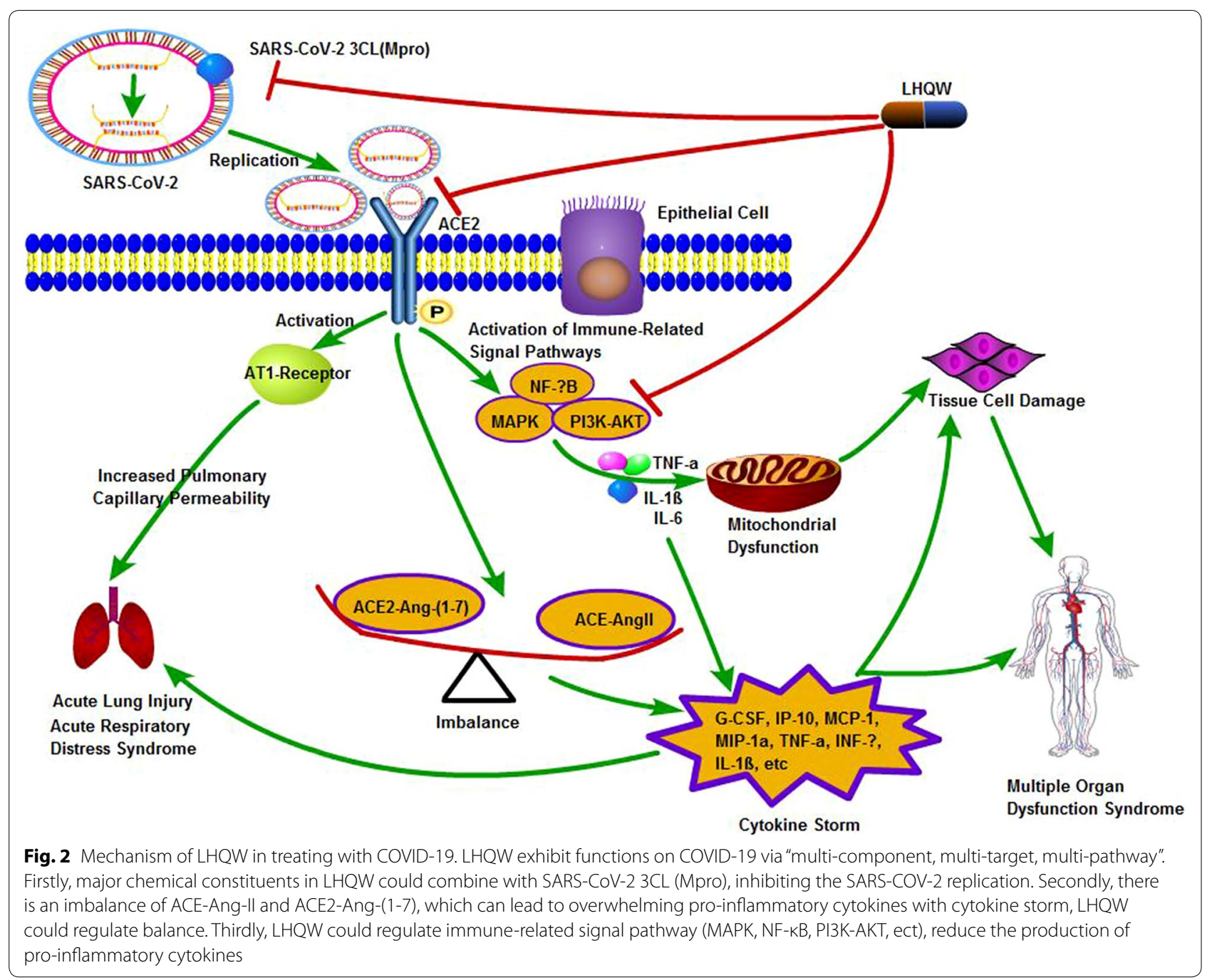

and more good messages about anti-SARS-CoV-2 activity of TCM will be discovered to benefit with COVID19 patients and finally overcome the current epidemic around the corner.

\footnotetext{
Abbreviations

COVID-19: Corona virus disease 2019; TCM: Traditional Chinese Medicine; CT: Computed tomography; ARDS: Acute respiratory distress syndrome; SARSCoV-2: Severe acute respiratory syndrome coronavirus 2; ACE2: Angiotensinconverting enzyme 2; MERS-CoV: Middle east respiratory syndrome coronavirus; NHC: National health commission; HXZQ: HuoxiangZhengqi; CK: Creatine kinase; LDH: Lactate dehydrogenase; TNF-a: Tumor necrosis factor a; LHQW: LianhuaQingwen; IL: Interleukin; CRP: c-reactive protein; PCT: Procalcitonin; SFJD: Shufeng Jiedu; AECOPD: Acute exacerbation of chronic obstructive pulmonary disease; ERK: Extracellular regulated protein kinases; TGF- $\beta$ : Transforming growth factor- $\beta$; NF-kB: Nuclear factor-kb; MAPK: Mitogen activated protein kinase; XBJ: Xuebijing: PI3K-AKT: Phosphatidylinositol 3-kinase protein kinase B; PTGS2: Prostaglandin-endoperoxide synthase 2; HSP90AB1: Heat shock protein $90 \mathrm{kDa}$ alpha B1; CAMSAP2: Calmodulin-regulated spectrinassociated proteins 2; HIF: Hypoxia inducible factor; VEGF: Vascular endothelial growth factor.
}

\section{Acknowledgements}

We would like to thank Dr. Xiao-Jun Feng and Lei Zhang for providing language help and writing assistance.

\section{Authors' contributions}

AS and SL designed the ideas; WN helped TT to collect and deal with the information; TT and YW wrote the manuscript. All authors read and approved the final manuscript.

\section{Funding}

This work was supported by the National Natural Science Foundation of China (No. 81803602) and research project commissioned by the Department of Pharmaceutical Administration of the National Health Commission of the People's Republic of China in 2018 (Grant No.: Pharmaceutical Administration [2018] No.2: A study on the commonality of drug use list of medical institutions at all levels).

\section{Availability of data and materials Not applicable.}

\section{Ethics approval and consent to participate} Not applicable.

\section{Consent for publication}

Not applicable. 


\section{Competing interests}

The authors declare that they have no competing interests.

Received: 17 March 2020 Accepted: 28 April 2020

Published online: 24 May 2020

\section{References}

1. Huang C, Wang Y, Li X, Ren L, Zhao J, Hu Y, et al. Clinical features of patients infected with 2019 novel coronavirus in Wuhan, China. Lancet. 2020;395(10223):497-506.

2. Li H, Lu CZ, Tang KC. Clinical observation on treatment of SARS with combination of chaihu droplet pill and huoxiangzhengqi droplet pill. Zhongguo Zhong Xi Yi Jie He Za Zhi. 2004;24:321-4.

3. Zhang Z, Li X, Zhang W, Shi ZL, Zheng Z, Wang T. Clinical features and treatment of 2019-nCov pneumonia patients in Wuhan: report of a couple cases. Virol Sin. 2020. https://doi.org/10.1007/s12250-020-00203-8.

4. Xu Z, Shi L, Wang Y, Zhang J, Huang L, Zhang C, et al. Pathological findings of COVID-19 associated with acute respiratory distress syndrome. Lancet Respir Med. 2020;8(4):420-2.

5. He YH, Zhao HY, Liu ZL, Lu C, Luo XJ, Lin SQ, et al. Effects of huoxiangzhengqi liquid on enteric mucosal immune responses in mice with Bacillus dysenteriae and Salmonella typhimurium induced diarrhea. World J Gastroenterol. 2006;12(45):7346-9.

6. Deng YJ, Liu BW, He ZX, Liu T, Zheng RL, Yang AD, et al. Study on active compounds from Huoxiang Zhengqi Oral Liquid for prevention of coronavirus disease 2019 (COVID-19) based on network pharmacology and molecular docking. Chin Tradit Herbal Drugs. 2020;51(5):1113-22.

7. Cheng DZ, Li Y. Clinical effectiveness and case analysis in 54 NCP patients treated with Lianhuaqingwen granules. World Chin Med. 2020;15(2):150-4.

8. Ling $X Y$, Tao JL, Sun $X$, Yuan B. Exploring material basis and mechanism of Lianhua Qingwen prescription against coronavirus based on network pharmacology. Chin Tradit Herbal Drugs. 2020;51(7):1723-30.

9. Zhang C, Wu Z, Li JW, Zhao H, Wang GQ. The cytokine release syndrome (CRS) of severe COVID-19 and Interleukin-6 receptor (IL-6R) antagonist Tocilizumab may be the key to reduce the mortality. Int J Antimicrob Agents. 2020. https://doi.org/10.1016/j.jiantimicag.2020.105954.

10. Lin L, Dai F, Ren G, Wei J, Chen Z, Tang X. Efficacy of lianhuaqingwen granules in the management of chronic rhinosinusitis without nasal polyps. Am J Otolaryngol. 2020;41(1):102311.

11. Li RF, Hou YL, Huang JC, Pan WQ, Ma QH, Shi YX, et al. Lianhuaqingwen exerts anti-viral and anti-inflammatory activity against novel coronavirus (SARS-CoV-2). Pharmacol Res. 2020. https://doi.org/10.1016/j. phrs.2020.104761.

12. Lin Q, Liao CX, Wei XH, Chen SY, Qian HJ. Therapeutic effect of LianhuaQingwen granule combined with sequential therapy of azithromycin on children mycoplasma pneumoniae pneumonia complicated with atelectasis and its effect on T lymphocyte subsets and inflammatory factors. Mod J of Integrated Tradi Chin Western Med. 2019;28(2):153-8.

13. Yao X, Cao LF, Yang J, Yao MX, Zhao L. Curative effect evaluation of ShufengJiedu Capsules for the treatment of acute exacerbation of chronic obstructive pulmonary disease. China J Tradit Chin Med Pharm. 2017;32(1):347-50.

14. Ji S, Bai Q, Wu X, Zhang DW, Wang S, Shen JL, et al. Unique synergistic antiviral effects of ShufengJiedu Capsule and oseltamivir in influenza a viral-induced acute exacerbation of chronic obstructive pulmonary disease. Biomed Pharmacother. 2020;121:109652.

15. Wang Z, Chen X, Lu Y, Chen F, Zhang W. Clinical characteristics and therapeutic procedure for four cases with 2019 novel coronavirus pneumonia receiving combined Chinese and Western medicine treatment. Biosci Trends. 2020;14(1):64-8.

16. Qu XK, Hao SL, Ma JH, Wei GY, Song KY, Tang C, et al. Observation on clinical effect of Shufeng Jiedu Capsule combined with Arbidol Hydrochloride Capsule in treatment of COVID-19. Chin Tradit Herbal Drugs. 2020;51(5):1167-70.

17. Tao Z, Gao J, Zhang G, Xue M, Yang W, Tong C, et al. ShufengJiedu capsule protect against acute lung injury by suppressing the MAPK/NF-KB pathway. Biosci Trends. 2014;8(1):45-51.

18. Shen F, Fu ZY, Wu YR, Kuang GY, Li L, Zhu KM, et al. The potential targets and mechanisms of Shufeng Jiedu Capsule for novel coronavirus pneumonia (COVID-19) based on network pharmacology and molecular docking. Guiding J Tradit Chin Med Pharm. 2020;26(5):8-15.

19. Duan LM, Ning CZ. Observation curative effect of Xuebijing injection synergisticin treatment of acute exacerbation of chronic obstructive pulmonary disease. China Contin Med Educ. 2015;7(31):219-20.

20. Peng YQ, Mao YM, Zhu JQ, Chen H, Qiu XL, Jiang JH, et al. A clinical study of short-term Xuebijing injection on treatment of patients with acute exacerbation of chronic obstructive pulmonary disease. Chin J Integrated Tradit West Med Intensive Crit Care. 2008;15(3):178-80.

21. He TM, Duan CC, Li XF, Zhang JY. Potential mechanism of Xuebijing injection in treatment of coronavirus pneumonia based on network pharmacology and molecular docking. Chin J Mod Appl Pharm. 2020;37(4):398-405

22. Sun MJ, Tu YR, OuYang HZ, Chang YX, He J. Effect of treatment with Xuebijing injection and its pharmacokinetics markers on serum inflammatory factor indude TNF-a, IL-1, IL-6, IL-8 and IL-10 in rats with sepsis. J Tianjin Univ Tradi Chin Med. 2018;37(1):13-5.

23. Zhang SW, Sun $C D$, Wen $Y$, Yin $C H$. Effect of treatment with Xuebijing injection on serum inflammatory mediators and Th1/2 of spleen in rats with sepsis. Zhongguo Wei Zhong Bing Ji Jiu Yi Xue. 2006;18(11):673-6.

24. Dai XG, Yao YM, Ai YH. Effect of apoptosis of CD4 + CD25 + regulatory T lymphocytes on polarization of helper T lymphocytes and potential interventional influence of Xuebijing injectionin septic rats. Zhongguo Wei Zhong Bing Ji Jiu Yi Xue. 2009;21(3):135-8.

25. Dai XG, Yao YM, Ai YH. Effect of Xuebijing injection on lipopolysaccharideinduced apoptosis of CD4 + CD25 + regulatory T cells and immune function of effector T cells in vitro. Chin J Emerg Med. 2009;18(9):932-6.

\section{Publisher's Note}

Springer Nature remains neutral with regard to jurisdictional claims in published maps and institutional affiliations.

Ready to submit your research? Choose BMC and benefit from

- fast, convenient online submission

- thorough peer review by experienced researchers in your field

- rapid publication on acceptance

- support for research data, including large and complex data types

- gold Open Access which fosters wider collaboration and increased citations

- maximum visibility for your research: over 100M website views per year

At BMC, research is always in progress.

Learn more biomedcentral.com/submissions 